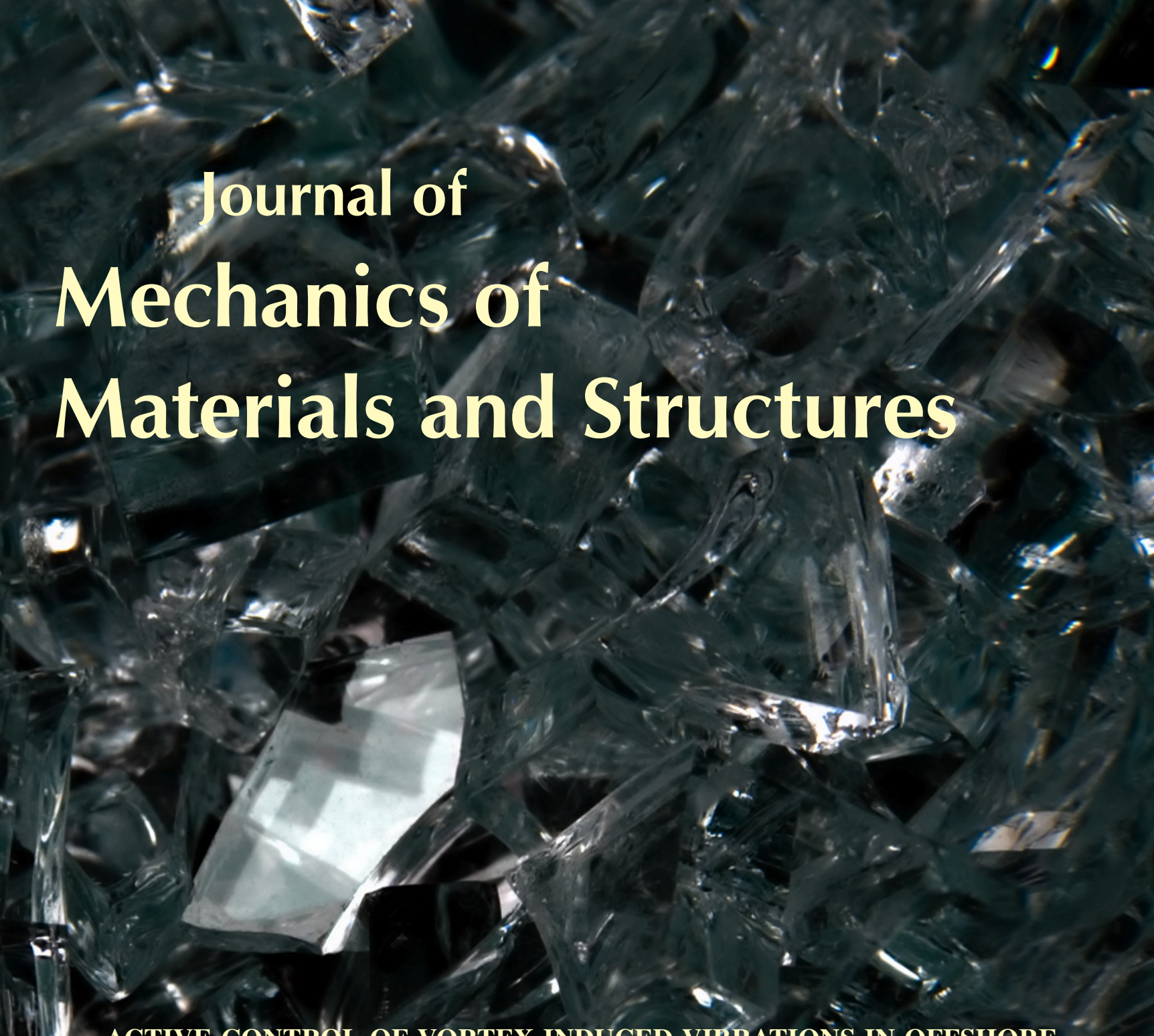
CATENARY RISERS: A NONLINEAR NORMAL MODE APPROACH

Carlòs E. N. Mazzilli and Césâr T. Sanches

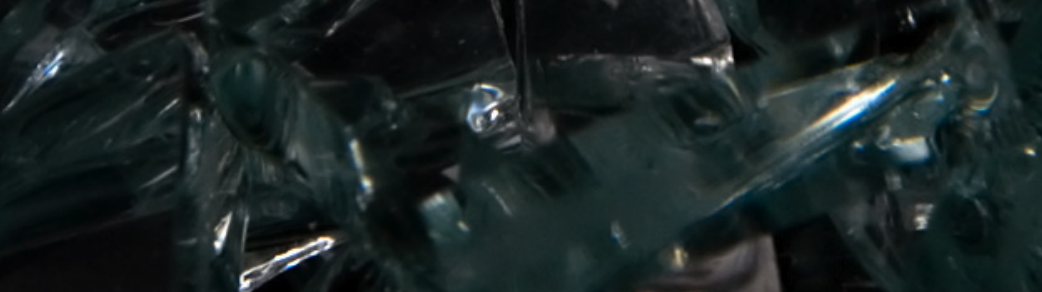

Volume 6, No. 7-8

Septembe October 2011

11) 5 mathematical sciences pi hlishers

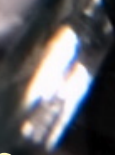




\title{
ACTIVE CONTROL OF VORTEX-INDUCED VIBRATIONS IN OFFSHORE CATENARY RISERS: A NONLINEAR NORMAL MODE APPROACH
}

\author{
CARlos E. N. MAZZilli AND CÉSAR T. SANChes
}

\begin{abstract}
Offshore catenary risers are used in the exploitation of deep-water oil and gas fields. They are subjected to severe dynamical loads, such as high-pressure inside-flow of fluids, sea-current external flow, and seawave motion of the floating production platform. This paper addresses the dynamic instability caused by vortex-induced vibrations (VIV). For simplicity, the touchdown-point motion and the mooring compliance are neglected in this introductory study. The nonlinear normal modes of a finite element model of the riser are determined, following the invariant manifold procedure, and a mode that is particularly prone to be excited by VIV is selected. A reduced mathematical model that couples the structural response and the fluid dynamics is used to foresee the vibration amplitudes when the instability caused by VIV takes over. Active control is introduced and the linear quadratic regulator is employed to determine gain matrices for the system and the observer. Results are compared with those from a linear analysis.
\end{abstract}

\section{Introduction}

The oil and gas industry has faced new challenges since several onshore and offshore fields have matured, aggravated by the growing global demand for energy and the volatility in oil prices. This scenario has led the operating companies to focus on deep and ultradeep water exploitation, bringing forth new concerns on reducing costs via advanced technological developments. Consequently, the offshore industry has increased its attention to new realms of research such as nonlinear dynamics of structures and computational fluid dynamics.

If subsea exploration has always been a complex and demanding activity, from now on, in view of the upcoming developments in deep and ultradeep water, it will be even more challenging. In fact, the offshore industry has already begun to explore in water depths at the limits of current technology and has plans to access depths over $2,500 \mathrm{~m}$. It is already developing subsea production systems in preparation for ultradeep water production that include more flexible and lighter risers that operate under harsher environmental conditions. From this standpoint, nonlinear effects are expected to play a major role in riser global dynamics.

The objective of this paper is to present a numerical study on the fluid-structure interaction and structural nonlinear dynamic behavior of a deep-water catenary riser subjected to in-plane vortex-induced vibration (VIV), employing the van der Pol oscillator proposed in [Blevins 1990] to represent the fluid dynamics and to the nonlinear modal analysis technique, respectively. A computational model is initially proposed using the finite element method. Geometrically nonlinear finite elements are employed so that the equations of motion take into consideration quadratic and cubic nonlinearities, expressed in terms

The first author acknowledges the support of CNPq under Grant 301942/2009-9.

Keywords: catenary risers, VIV, nonlinear modes, finite element method, active control. 
of third and fourth-order tensors, respectively. These nonlinear equations allow for the assessment of both cable and beam behavior. In this work, the motion of the floating production unit (FPU) and the unilateral contact at the touchdown zone (TDZ) have been neglected. Hence, the catenary riser has been modeled with fixed pinned ends, just allowing for rotation. Further advances, regarding a numerical approach for the TDZ, are under development, since considerable programming effort is required to take into consideration contact at the TDZ. It is worth mentioning that an analytical study of the local dynamics of steel catenary risers (SCR), considering unilateral contact at the TDZ, led to an approximate evaluation of the associated nonlinear normal modes of vibration [Mazzilli and Lenci 2008]. Nonlinear modes seem to play an important role in the structural representation of deep-water risers by models with small numbers of degrees of freedom. A free-hanging catenary is addressed in a case study, considering a certain nonlinear normal mode of vibration, which is seen to be relevant for the analysis of the VIV motion. The ultimate goal is to develop a coupled fluid-riser low-dimensional model, to which active control can be added in a simple way.

\section{Nonlinear modes}

This paper should be regarded as an initial effort to assess the nonlinearly coupled fluid-riser interaction. The riser will be modeled as a plane frame with geometric nonlinearities due to the coupling of tangential and transversal displacements. Material linearity will be assumed. Torsion and 3D effects, as well as FPU motion and unilateral contact at the TDZ, will be neglected.

Although internal resonance may come into play, which would require the consideration of the socalled nonlinear multimodes, the paper will concentrate on the nonlinear normal modes.

It is believed that the nonlinear normal modes and multimodes may play an important role in generating reliable models with few degrees of freedom, still keeping the essential behavior of risers under different sea-loading conditions, such as in cases of high-frequency VIV and low-frequency drifting.

2.1. FEM formulation. Typically, the equations of motion of a general $n$-degree of freedom finite element model of an elastic plane frame with geometric nonlinearities under free vibrations read [Soares and Mazzilli 2000]:

$$
M_{r s} \ddot{p}_{s}+D_{r s} \dot{p}_{s}+K_{r s} p_{s}=0, \quad r, s=1, \ldots, n,
$$

where Einstein's convention for summation is employed; $p_{s}$ are the generalized coordinates. The matrices of mass $M_{r s}$, equivalent damping $D_{r s}$, and stiffness $K_{r s}$ depend on the generalized coordinates and velocities as follows:

$$
\begin{aligned}
M_{r s} & ={ }^{0} M_{r s}+{ }^{1} M_{r s}^{i} p_{i}+{ }^{2} M_{r s}^{i j} p_{i} p_{j}, \\
D_{r s} & ={ }^{0} D_{r s}+{ }^{1} D_{r s}^{i} \dot{p}_{i}+{ }^{2} D_{r s}^{i j} \dot{p}_{i} p_{j}, \\
K_{r s} & ={ }^{0} K_{r s}+{ }^{1} K_{r s}^{i} p_{i}+{ }^{2} K_{r s}^{i j} p_{i} p_{j},
\end{aligned}
$$

where ${ }^{0} M_{r s},{ }^{1} M_{r s}^{i},{ }^{2} M_{r s}^{i j},{ }^{0} D_{r s},{ }^{1} D_{r s}^{i},{ }^{2} D_{r s}^{i j},{ }^{0} K_{r s},{ }^{1} K_{r s}^{i}$, and ${ }^{2} K_{r s}^{i j}(r, s, i, j=1, \ldots, n)$ are constants.

2.2. Linear and nonlinear modes. During a modal motion, the phase trajectories of a discretized linear system remain confined to a $2 \mathrm{D}$ eigenplane, in much the same way as the phase trajectory of a onedegree-of-freedom system with generalized coordinate $x$ remains confined to the plane $x \times \dot{x}$. Due to this invariance property, such an eigenplane is an invariant manifold of the dynamical system. 
In nonlinear systems the invariant manifolds are no longer planes, and the motions whose trajectories are confined to them are called nonlinear normal modes. Normally, there are $n$ invariant manifolds, each one corresponding to a different mode; these manifolds contain the equilibrium point at which they are tangent to the eigenplanes of the linearized system.

Such a geometric characterization of a modal motion suggests the so-called invariant-manifold procedure to determine normal modes, which was proposed in [Shaw and Pierre 1993] and applied to systems of few degrees of freedom. In [Soares and Mazzilli 2000] the procedure was extended to full finite element models of plane frames.

An alternative technique to evaluate nonlinear normal modes of finite element models was proposed in [Mazzilli and Baracho Neto 2002], based on the method of multiple scales.

To handle cases of coupled modal motions of nonlinear systems subjected to internal resonance, the multiple-scales procedure has also been successfully extended in [Baracho Neto and Mazzilli 2005]. Here, the ensuing vibration takes place in an invariant manifold embedded in the phase space, whose dimension is twice the number of the normal modes that interact. This manifold contains a stable equilibrium point at which it is tangent to the subeigenspace of the linearized system, which characterizes the corresponding coupled linear modes. On this manifold, the system behaves like an $M$-degree of freedom oscillator, where $M$ is the number of coupled normal modes.

2.3. Invariant manifold procedure. Here, the fundamental steps of the invariant-manifold procedure are followed [Shaw and Pierre 1993], keeping in mind its application to finite element models of risers.

Introducing the notation $x_{i}=p_{i}$ and $y_{i}=\dot{p}_{i}=\dot{x}_{i}$, the system (1) can be written in first-order form as

$$
\dot{x}_{i}=y_{i}, \quad \dot{y}_{i}=f_{i}\left(x_{1}, \ldots, x_{n}, y_{1}, \ldots, y_{n}\right), \quad i=1, \ldots, n .
$$

Series expansions for the functions $f_{i}\left(x_{1}, \ldots, x_{n}, y_{1}, \ldots, y_{n}\right)$ in the neighborhood of the equilibrium point are introduced in (4):

$$
\begin{gathered}
f_{i}\left(x_{1}, \ldots, x_{n}, y_{1}, \ldots, y_{n}\right)=B_{i j} x_{j}+C_{i j} y_{j}+E_{i j m} x_{j} x_{m}+F_{i j m} x_{j} y_{m}+G_{i j m} y_{j} y_{m} \\
+H_{i j m p} x_{j} x_{m} x_{p}+L_{i j m p} x_{j} x_{m} y_{p}+N_{i j m p} x_{j} y_{m} y_{p}+R_{i j m p} y_{j} y_{m} y_{p},
\end{gathered}
$$

where $B_{i j}, C_{i j}, E_{i j m}, F_{i j m}, G_{i j m}, H_{i j m p}, L_{i j m p}, N_{i j m p}$, and $R_{i j m p}(i, j, m, p=1, \ldots, n)$ are known constants that depend on the previously introduced ${ }^{0} M_{r s},{ }^{1} M_{r s}^{i},{ }^{2} M_{r s}^{i j},{ }^{0} D_{r s},{ }^{1} D_{r s}^{i},{ }^{2} D_{r s}^{i j},{ }^{0} K_{r s},{ }^{1} K_{r s}^{i}$, and ${ }^{2} K_{r s}^{i j}(r, s, i, j=1, \ldots, n)$, as detailed in [Soares and Mazzilli 2000].

If, during a modal motion, the trajectory of the solution in the phase-space is restricted to a $2 \mathrm{D}$ surface, then it must be possible to express each generalized displacement or velocity as a function of two of them, for instance $u=x_{k}$ and $v=y_{k}$, for a certain degree of freedom $k$, at least in the neighborhood of the equilibrium point.

By substituting the expressions

$$
x_{i}(t)=X_{i}(u(t), v(t)), \quad y_{i}(t)=Y_{i}(u(t), v(t)), \quad i=1, \ldots, n,
$$

in (3), one arrives at

$$
\begin{aligned}
& \frac{\partial X_{i}}{\partial u} v+\frac{\partial X_{i}}{\partial v} f_{k}\left(X_{1}, \ldots, X_{n}, Y_{1}, \ldots, Y_{n}\right)=Y_{i} \\
& \frac{\partial Y_{i}}{\partial u} v+\frac{\partial Y_{i}}{\partial v} f_{k}\left(X_{1}, \ldots, X_{n}, Y_{1}, \ldots, Y_{n}\right)=f_{i}\left(X_{1}, \ldots, X_{n}, Y_{1}, \ldots, Y_{n}\right), \quad i=1, \ldots, n,
\end{aligned}
$$


which is a nonlinear system of partial differential equations having the functions $X_{i}$ and $Y_{i}$ as unknowns, which may be as difficult to solve as the original equations. However, if we look for an approximate solution, these functions can be written as polynomials up to cubic terms:

$$
\begin{gathered}
X_{i}(u, v)=a_{1 i} u+a_{2 i} v+a_{3 i} u^{2}+a_{4 i} u v+a_{5 i} v^{2}+a_{6 i} u^{3}+a_{7 i} u^{2} v+a_{8 i} u v^{2}+a_{9 i} v^{3}, \\
Y_{i}(u, v)=b_{1 i} u+b_{2 i} v+b_{3 i} u^{2}+b_{4 i} u v+b_{5 i} v^{2}+b_{6 i} u^{3}+b_{7 i} u^{2} v+b_{8 i} u v^{2}+b_{9 i} v^{3},
\end{gathered}
$$

where $a_{j i}$ and $b_{j i}(j=1, \ldots, 9$ and $i=1, \ldots, n)$ are constants to be determined.

Now, if we substitute (7) and (4) in (6), a system of nonlinear polynomial equations having the $a$ 's and $b$ 's as unknowns is formed. In general, there are $n$ solutions to this system, each one corresponding to a different set of modal relations (5), that is, a different invariant manifold. Moreover, substituting any one of these solutions in (7) and the resulting expressions in (5), the $k$-th equation in (3)-(4) - called the modal oscillator equation - characterizes the dynamics of the corresponding mode.

Details of the procedure just outlined are avoided here for brevity, but can be found in [Soares and Mazzilli 2000], where it is also shown that the solution of the system of nonlinear polynomial equations mentioned above can be avoided, provided the eigenvalues and eigenvectors of the linearized system are known.

\section{Fluid-structure interaction}

Among the possible scenarios for the fluid-structure interaction, the case of vortex-induced vibrations (VIV) is here addressed [Williamson and Govardhan 2004]. In the subcritical regime, the flow with freestream velocity $U=U_{\infty}$ around a circular cylinder of diameter $D$ forms a von Kármán vortex street as the one shown in Figure 1 [Assi 2009]. The Strouhal number, St, is the predominant frequency of vortex shedding $f_{s}$ multiplied by the cylinder diameter $D$ and divided by the free-stream velocity:

$$
\mathrm{St}=\frac{f_{s} D}{U} \text {. }
$$
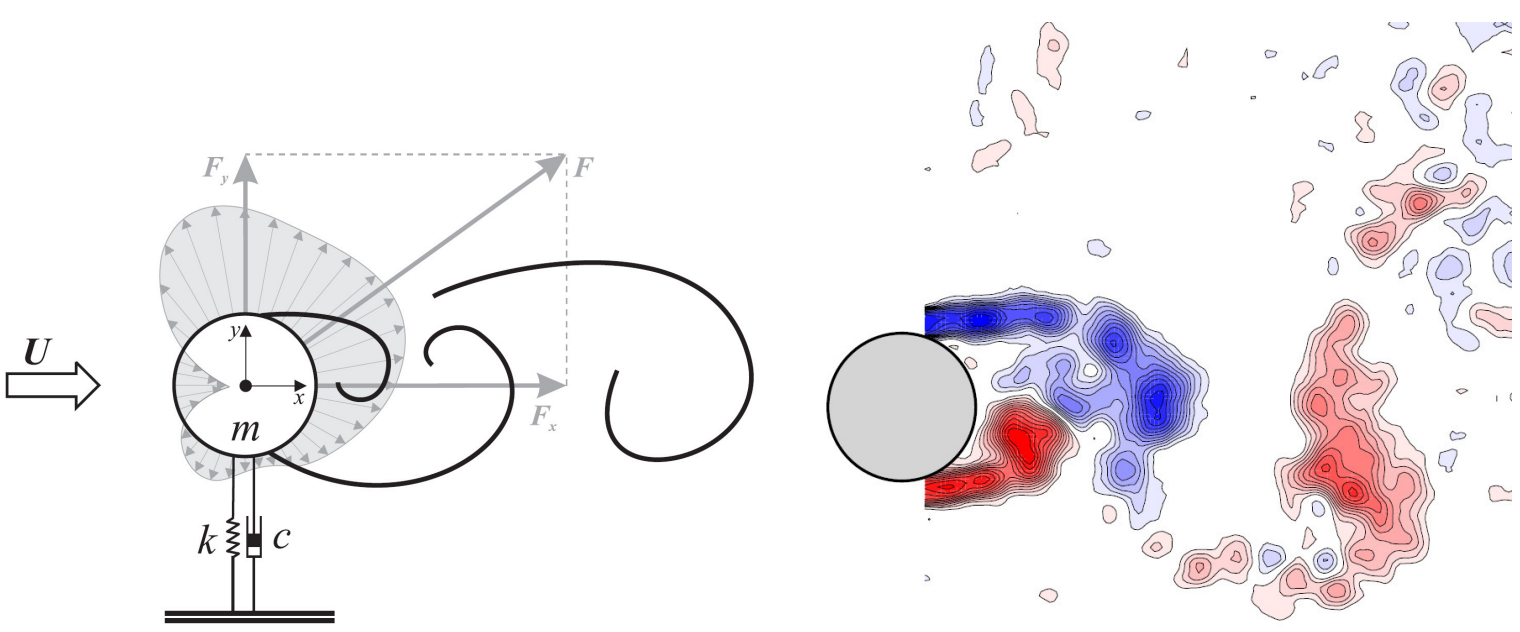

Figure 1. Visualization of von Kármán vortex street [Assi 2009]. 
In the subcritical regime, the Reynolds number, Re, based on the cylinder diameter is in the range $200 \leq \operatorname{Re} \leq 5 \times 10^{5}$. In this range, experimental results found in the literature indicate that the Strouhal number is almost constant $\mathrm{St}=0.20$ and the drag coefficient is $C_{d}=1.2$.

A classical approach to characterizing the dynamics of the coupled fluid-structure system [Facchinetti et al. 2004] is to employ phenomenological models, a thorough review of which can be found in [Gabbai and Benaroya 2005]. In this paper a very simple phenomenological model based in [Blevins 1990] has been considered, leading to the following system of differential equations, the first of which refers to the nonlinear dynamics of the reduced-order model of the structural system and the second of which to the fluid dynamics:

$$
\begin{gathered}
\frac{d^{2} u}{d t^{2}}+2 \omega_{n}\left(\zeta_{s}+\zeta_{F}\right) \frac{d u}{d t}+\omega_{n}^{2} u+\text { nonlinear terms }=\left(\frac{\rho D^{2}}{m}\right) \frac{U}{D} a_{4} \frac{d w}{d t}, \\
\frac{d^{2} w}{d t^{2}}+\omega_{s}^{2} w=\left(\frac{a_{1}-a_{4}}{a_{0}}\right) \frac{U}{D} \frac{d w}{d t}-\frac{a_{2}}{a_{0}} \frac{1}{U D}\left(\frac{d w}{d t}\right)^{3}+\frac{a_{4}}{a_{0}} \frac{U}{D} \frac{d u}{d t},
\end{gathered}
$$

where $u$, as before, is the modal generalized coordinate, $\omega_{n}$ is the linear natural frequency of the chosen vibration mode, $\zeta_{s}$ is the structural damping ratio, $\zeta_{F}$ is the fluid damping ratio, $\rho$ is the seawater specific mass, $m$ is the modal mass including both the structure and the fluid added mass, $w$ is the fluid hidden variable [Blevins 1990], $\omega_{s}=2 \pi f_{s}$ is the vortex shedding frequency, and $a_{0}, a_{1}, a_{2}$, and $a_{4}$ are known constants.

In this paper, (9) is sought by using the invariant manifold approach. Therefore, second and third-order nonlinear terms will arise as a consequence of the nonlinear structural formulation.

\section{Case study: Part A}

Table 1 presents the riser data used to model the structural system. Figure 2 shows the riser finite element model with 77 degrees of freedom and 26 nonlinear Bernoulli-Euler-based elements. The reduced number of elements used is due to the considerable computational effort required to work out third and fourth-order tensors that led this FEM model to allocate approximately 2 GB of RAM. It took 17 hours of processing time using a $1.6 \mathrm{GHz}$ processor to obtain the nonlinear normal modes of the system.

Young's modulus

Riser length

Cross-section area

Cross-section moment of inertia

Riser external diameter

Riser thickness

Initial tension (at the top)

Initial tension (at the bottom)

Riser mass per unit length (water inside and added mass)

Riser weight per unit length
$E=2.1 \times 10^{11} \mathrm{~N} / \mathrm{m}^{2}$

$l=1,800 \mathrm{~m}$

$A=1.1021 \times 10^{-2} \mathrm{~m}^{2}$

$I=4.72143 \times 10^{-5} \mathrm{~m}^{4}$

$D=2.032 \times 10^{-1} \mathrm{~m}$

$e=19.05 \mathrm{~mm}$

$T_{0 t}=2 \times 10^{6} \mathrm{~N}$

$T_{0 b}=6.914 \times 10^{5} \mathrm{~N}$

$m=108 \mathrm{~kg} / \mathrm{m}$

$p=727 \mathrm{~N} / \mathrm{m}$

Table 1. Typical steel riser data. 


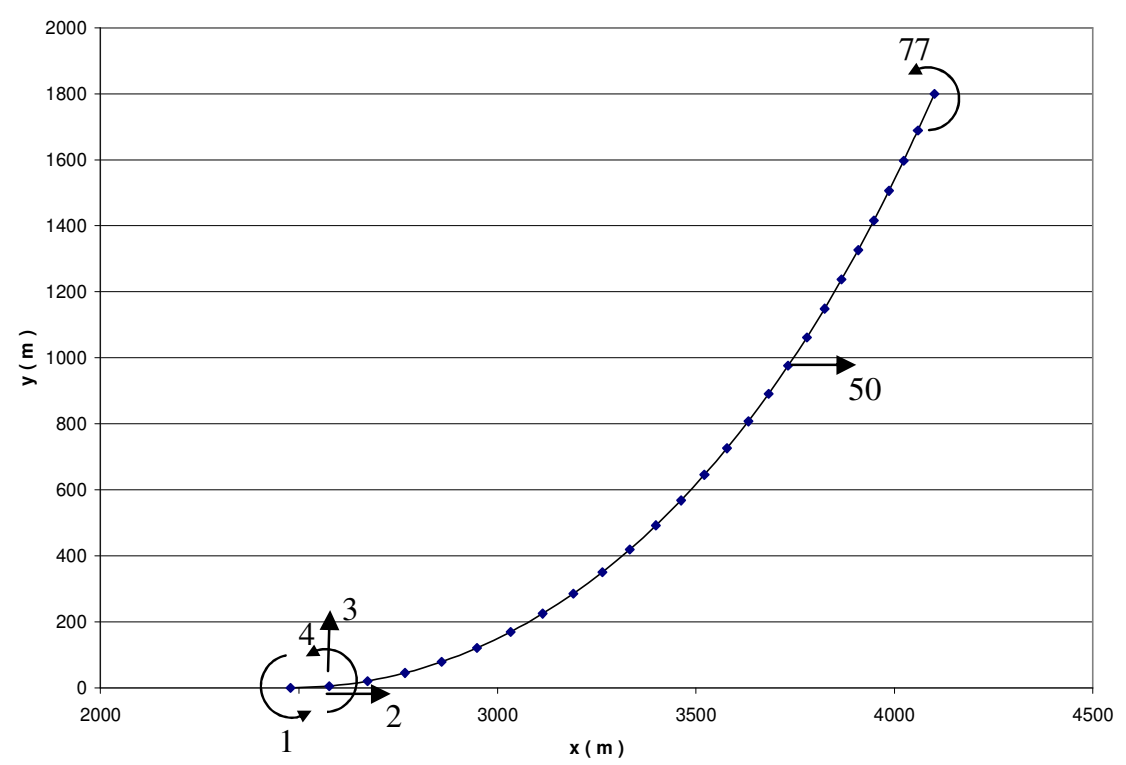

Figure 2. Riser finite element model and the chosen modal variable $u=p_{50}$.

VIV calculations were performed assuming the Strouhal number to be $\mathrm{St}=0.20$ and the free-stream velocity $U=0.5 \mathrm{~m} / \mathrm{s}$ (normal to the motion plane). Taking this into account, the vortex-shedding frequency approaches the natural frequency of the 26th vibration mode. Therefore, it is assumed that the lock-in occurs for this mode. As a result, the following system of equations is sought to represent the dynamics of the coupled fluid-structure system herein addressed:

$$
\begin{gathered}
\ddot{u}+8.1921 u+39 v+22.16 u^{2}-3.0673 v^{2}-70.823 u^{3}+533.54 u v^{2}=38.95 \dot{w}, \\
\ddot{w}+9.8696 w=4.17 \dot{w}^{3}+0.3125 \dot{w}+1.98 v,
\end{gathered}
$$

where $u$ and $v$ are respectively the modal displacement and the modal velocity, so $\dot{u}=v$. The chosen modal displacement is $u=p_{50}$, as shown in Figure 2.

In Figure 3, a phase portrait, for both "linear" and nonlinear responses, can be observed. The "linear" response is the response of the coupled fluid-structure system when only the structural system is linearized. Thus, the dynamics of the fluid remain nonlinear even when the linear structural system is considered. The total nonlinear amplitude amounts to $0.565 \mathrm{~m}$ whilst the "linear" response leads to a total amplitude of $0.567 \mathrm{~m}$. Although there is no noteworthy difference in the total amplitudes, it's interesting to stress that the nonlinear amplitude extremes are different with the maximum of $0.3057 \mathrm{~m}$ and the minimum of $-0.2596 \mathrm{~m}$. On the other hand, the "linear" system gives the same absolute values for the extremes $(0.2834 \mathrm{~m}$ and $-0.2834 \mathrm{~m})$. Hence, the nonlinear system is able to capture the asymmetric stiffness, due to the riser static curvature.

\section{Active control}

The Luenberger observer [Luenberger 1979] is employed in what follows. Here, only one specific nonlinear normal mode will be considered when modeling the controlled system. Therefore, this should 


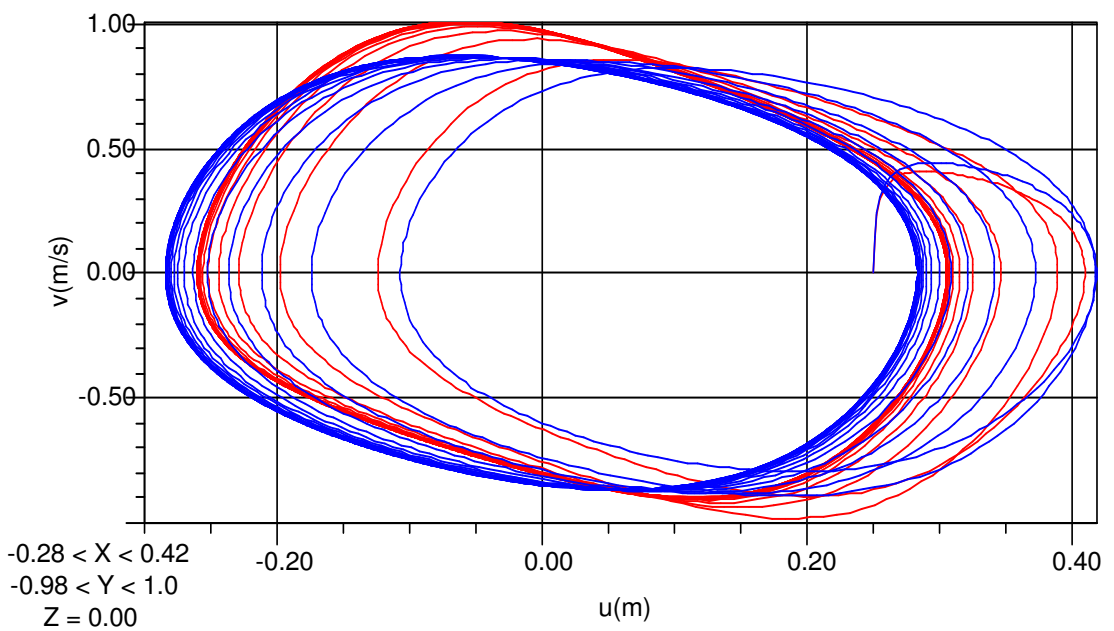

Figure 3. Phase portrait ("linear" in blue, nonlinear in red).

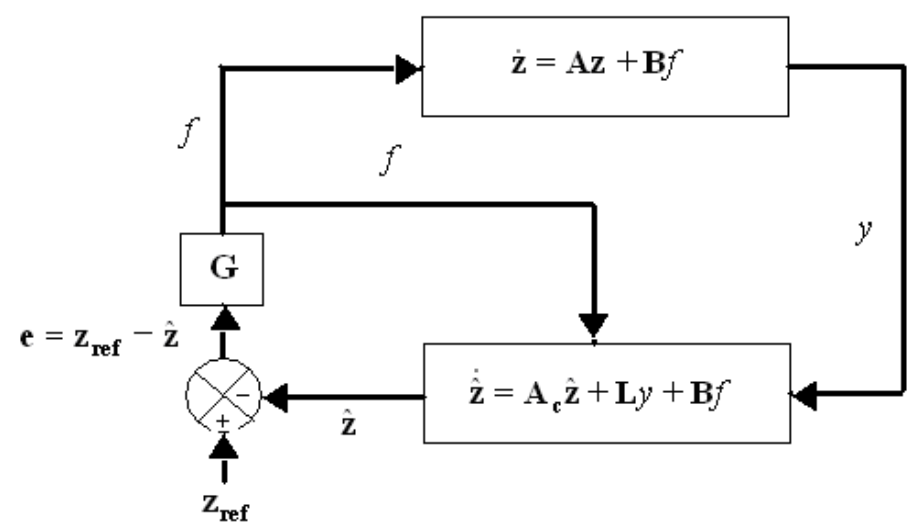

Figure 4. The control system.

be regarded as an initial investigation of the behavior of a SCR riser under VIV. Emphasis is placed on the system design, namely the system and the observer gain matrices. Considerations regarding actuators, sensors, or physical installations are not within the scope of this work. A simple example regarding controlled VIV will be addressed in Section 6. Although the structural system will be modeled as nonlinear, the employed observer will still be assumed to be linear. A nonlinear observer would lead to a much more complex model, but the control system would present a larger stability window. However, it will be seen that the linear observer already leads to stable responses, even when large amplitudes and considerable nonlinear effects are taken into consideration. Optimal control is employed via the linear quadratic regulator [Ogata 1995; Preumont 2002]. Figure 4 represents the control system.

The matrix equation of the structural system with the actuator term can be written as

$$
\dot{z}=\boldsymbol{A} z+B f,
$$

where $z=\left\{\{\tilde{\boldsymbol{x}}\}^{T}\{\dot{\tilde{\boldsymbol{x}}}\}^{T}\right\}^{T}$ is the $2 n \times 1$ phase-space vector for a $n$-degree of freedom system, $\tilde{\boldsymbol{x}}$ is the $n \times 1$ 
vector of principal generalized coordinates in the configuration space, $f=\boldsymbol{G}\left(\boldsymbol{z}_{\mathrm{ref}}-\hat{\boldsymbol{z}}\right)$ is the input control force, $\boldsymbol{G}$ is the $1 \times 2 n$ system gain matrix, and $\boldsymbol{A}=\boldsymbol{A}(\boldsymbol{z})$ is the $2 n \times 2 n$ nonlinear system matrix. Let $\boldsymbol{T}$ be the $n \times n$ linear eigenvector matrix of the structural system and $\boldsymbol{b}=\boldsymbol{T}^{T} \boldsymbol{n}$ an $n \times 1$ vector, where $\boldsymbol{n}$ is the $n \times 1$ actuator position vector. Hence, the $2 n \times 1$ vector $\boldsymbol{B}=\left\{\{\boldsymbol{b}\}^{T}\{\boldsymbol{0}\}^{T}\right\}^{T}$ is defined.

The system of differential equations presented in Figure 4 can be rewritten as follows:

$$
\left\{\begin{array}{c}
\dot{z} \\
\dot{\hat{z}}
\end{array}\right\}=\left[\begin{array}{cc}
\boldsymbol{A} & -\boldsymbol{B} \boldsymbol{G} \\
\boldsymbol{L} \boldsymbol{C} & \boldsymbol{A}_{c}-\boldsymbol{B} \boldsymbol{G}
\end{array}\right]\left\{\begin{array}{l}
z \\
\hat{z}
\end{array}\right\}+\left\{\begin{array}{l}
\boldsymbol{B} \\
\boldsymbol{B}
\end{array}\right\} f_{\mathrm{ref}},
$$

where $\boldsymbol{A}_{c}=\boldsymbol{A}-\boldsymbol{L} \boldsymbol{C}, f_{\text {ref }}$ is an arbitrary force related to an arbitrary state vector $\boldsymbol{z}_{\text {ref }}$, and $\boldsymbol{L}$ is the $2 n \times 1$ observer gain matrix. Defining the $1 \times 2 n$ matrix $\boldsymbol{C}=\left\{\begin{array}{ll}\boldsymbol{c} & \mathbf{0}\end{array}\right\}$, where $\boldsymbol{c}=\boldsymbol{h}^{T} \boldsymbol{T}$ is a $1 \times n$ matrix and $\boldsymbol{h}$ is the $n \times 1$ sensor position vector, then $y=\boldsymbol{C} \boldsymbol{z}$. For further details see [Ogata 1995]. The solution of (14) is pursued via the Runge-Kutta method.

5.1. Linear quadratic regulator. A linear state feedback with constant system gain $\boldsymbol{G}$ is sought, such that the following quadratic cost functional is minimized:

$$
\min J=\int_{0}^{\infty}\left(\frac{1}{2} \boldsymbol{z}^{T} \boldsymbol{Q} z+\frac{1}{2} f^{T} \boldsymbol{R} f\right) \mathrm{d} t, \quad \text { such that } \dot{\boldsymbol{z}}=\boldsymbol{A} \boldsymbol{z}+\boldsymbol{B} f
$$

where $\boldsymbol{Q}$ is semipositive definite and $\boldsymbol{R}$ is strictly positive definite. The matrices $\boldsymbol{Q}$ and $\boldsymbol{R}$ are, at first, unknown and should be calibrated according to experimental results [Preumont 2002]. It is possible to show that the system gain matrix ends up being

$$
\boldsymbol{G}=\boldsymbol{R}^{-1} \boldsymbol{B}^{T} \boldsymbol{P}
$$

where $\boldsymbol{P}$ is a symmetric positive definite matrix that can be obtained from Riccati's equation:

$$
\boldsymbol{Q}+\boldsymbol{A}^{T} \boldsymbol{P}+\boldsymbol{P} \boldsymbol{A}-\boldsymbol{P} \boldsymbol{B} \boldsymbol{R}^{-1} \boldsymbol{B}^{T} \boldsymbol{P}=\mathbf{0} .
$$

\section{Case study: Part B}

To provide an introductory example of a forced controlled system, the coupled structural and fluid equations (accordingly to Section 3) for a SCR are written as follows:

$$
\begin{gathered}
\ddot{u}+8.1921 u+39 v+22.216 u^{2}-3.0673 v^{2}-70.823 u^{3}+533.54 u v^{2}=38.95 \dot{w}+f_{u}, \\
\ddot{w}+9.8696 w=0.3125 \dot{w}+4.17 \dot{w}^{3}+1.98 v,
\end{gathered}
$$

where $u$ is the chosen modal displacement, $v=\dot{u}$ the respective modal velocity, $f_{u}$ the actuator modal force, and $w$ the fluid hidden variable. As before, the data for the SCR are from Table 1 and Figure 2 depicts the finite element model. The nonlinear modal oscillator, (18), was obtained via the invariant manifold technique. It corresponds to the 26th nonlinear vibration mode. The control influence is introduced in the system as, mainly, a variation in the riser tension. As seen in Section 4, the simulation of the uncontrolled system, considering the nonlinear structural behavior, leads to a maximum displacement of $0.3057 \mathrm{~m}$ and a minimum displacement of $-0.2596 \mathrm{~m}$. The time response and the phase diagram for the controlled system are presented in Figure 5, from which it is seen that the maximum displacement for the controlled system, considering the nonlinear structural behavior, is $0.2571 \mathrm{~m}$ and the minimum is 


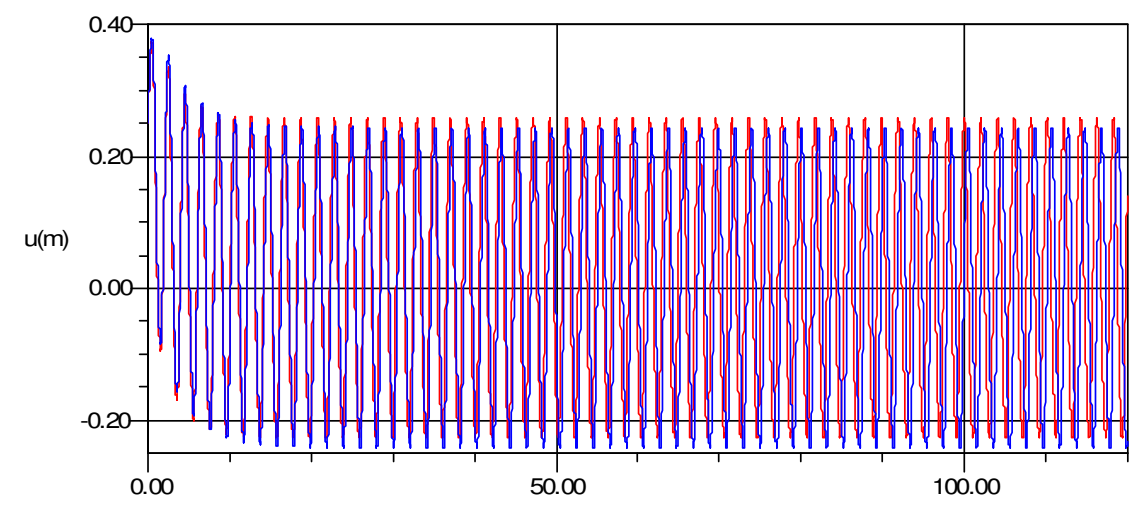

$0.00<\mathrm{X}<1.20 \mathrm{e}+2 ;-0.24<\mathrm{Y}<0.38$

$t(s)$

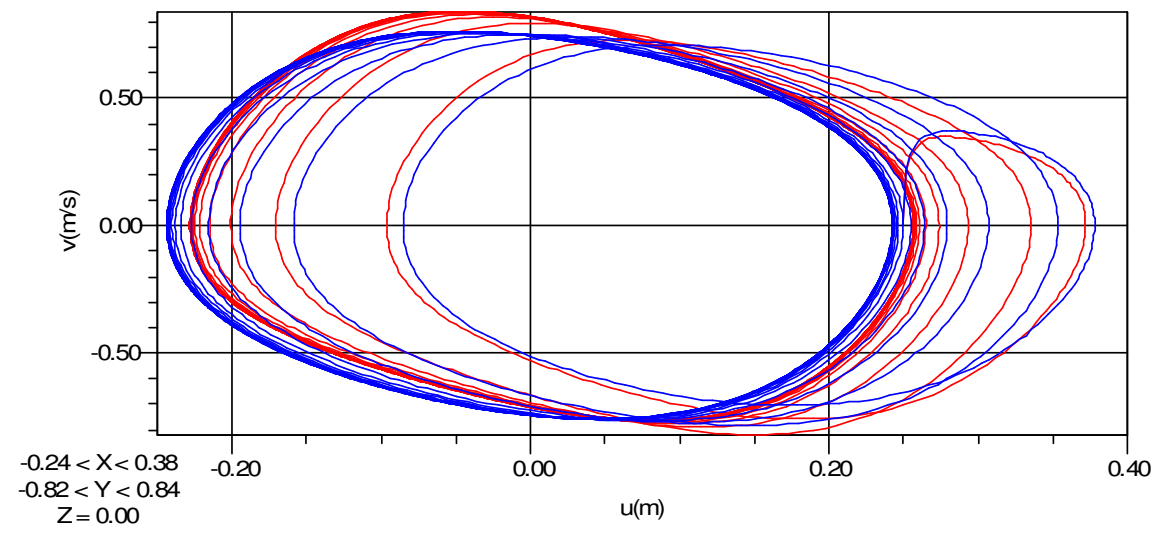

Figure 5. SCR time response and phase portrait (blue for linear and red for nonlinear).

$-0.2277 \mathrm{~m}$. Therefore, the amplitude of the controlled system is $14.16 \%$ smaller than the uncontrolled, but in order to reach such an amplitude reduction, an increase of approximately $50 \%$ in the riser tension is seen to be necessary. Consequently, it is questionable if the adoption of the riser tension as a control parameter is suitable for SCRs. It could be further argued that the tension variation for control purposes would affect the riser natural frequencies, so that the vortex shedding frequency might be detuned with respect to the previously locked-in mode, but could be tuned to another nearby mode. Of course, this behavior cannot be detected by the single-degree of freedom reduced-order model considered here and would require a more realistic analysis [Silveira et al. 2007; Josefsson and Dalton 2010].

\section{Conclusion}

We introduce tools of nonlinear dynamics, such as nonlinear normal modes and reduced-order modeling, based on which the analysis of offshore risers, considering geometrical nonlinearities, vortex-induced vibrations, and active control, may be pursued in a simple way. The case study addresses the response of a SCR, revealing not only remarkable quantitative differences in the estimates of maximum amplitudes between linear and nonlinear, uncontrolled and controlled models, but also qualitatively distinct behavior, due to the nonlinear effect of the riser statical curvature. 


\section{References}

[Assi 2009] G. R. S. Assi, Mechanisms for flow-induced vibration of interfering bluff bodies, Ph.D., Imperial College, Department of Aeronautics, London, 2009, available at http://www.ndf.poli.usp.br/ gassi/GAssi_PhD_2009.pdf.

[Baracho Neto and Mazzilli 2005] O. G. P. Baracho Neto and C. E. N. Mazzilli, "Evaluation of multi-modes for finite-element models: systems tuned into 1:2 internal resonance", Int. J. Solids Struct. 42:21-22 (2005), 5795-5820.

[Blevins 1990] R. D. Blevins, Flow-induced vibration, 2nd ed., Van Nostrand Reinhold, New York, 1990.

[Facchinetti et al. 2004] M. L. Facchinetti, E. de Langre, and F. Biolley, "Coupling of structure and wake oscillators in vortexinduced vibrations", J. Fluids Struct. 19:2 (2004), 123-140.

[Gabbai and Benaroya 2005] R. D. Gabbai and H. Benaroya, "An overview of modeling and experiments of vortex-induced vibration of circular cylinders", J. Sound Vib. 282:3-5 (2005), 575-616.

[Josefsson and Dalton 2010] P. M. Josefsson and C. Dalton, "An analytical/computational approach in assessing vortex-induced vibration of a variable tension riser", J. Offshore Mech. Arct. Eng. 132:3 (2010), article ID 031302-1/7.

[Luenberger 1979] D. G. Luenberger, Introduction to dynamic systems: theory, models, and applications, Wiley, New York, 1979.

[Mazzilli and Baracho Neto 2002] C. E. N. Mazzilli and O. G. P. Baracho Neto, "Evaluation of non-linear normal modes for finite-element models", Comput. Struct. 80:11 (2002), 957-965.

[Mazzilli and Lenci 2008] C. E. N. Mazzilli and S. Lenci, "Normal vibration modes of a slender beam on elastic foundation with unilateral contact", in XXII ICTAM (Adelaide, Australia, 2008), IUTAM - International Union of Theoretical and Applied Mechanics, 2008.

[Ogata 1995] K. Ogata, Discrete-time control system, Prentice Hall, Englewood Cliffs, 1995.

[Preumont 2002] A. Preumont, Vibration control of active structures: an introduction, Kluwer, Dordrecht, 2002.

[Shaw and Pierre 1993] S. W. Shaw and C. Pierre, "Normal modes for non-linear vibratory systems", J. Sound Vib. 164:1 (1993), 85-124.

[Silveira et al. 2007] L. M. Y. Silveira, C. M. Martins, L. D. Cunha, and C. P. Pesce, "An investigation on the effect of tension variation on the VIV of risers", in Proceedings of the 26th International Conference on Offshore Mechanics and Arctic Engineering, San Diego, 2007.

[Soares and Mazzilli 2000] M. E. S. Soares and C. E. N. Mazzilli, "Nonlinear normal modes of planar frames discretised by the finite element method", Comput. Struct. 77:5 (2000), 485-493.

[Williamson and Govardhan 2004] C. H. K. Williamson and R. Govardhan, "Vortex-induced vibrations", Annu. Rev. Fluid Mech. 36 (2004), 413-455.

Received 16 Jun 2010. Revised 2 Dec 2010. Accepted 17 Jan 2011.

CARlos E. N. MazziLli: cenmazzi@usp.br

Department of Structural and Geotechnical Engineering, Polytechnic School, University of São Paulo,

Av. Prof. Almeida Prado, trav. 2 n. 83, 05508-900 São Paulo-SP, Brasil

CÉSAR T. SANCHES: cesar.sanches@gmail.com

Department of Structural and Geotechnical Engineering, Polytechnic School, University of São Paulo,

Av. Prof. Almeida Prado, trav. 2 n. 83, 05508-900 São Paulo-SP, Brasil 


\title{
JOURNAL OF MECHANICS OF MATERIALS AND STRUCTURES
}

\author{
jomms.org
}

Founded by Charles R. Steele and Marie-Louise Steele

EDITORS

Charles R. SteEle

DAVIDE BIGONI

Stanford University, USA

YASUHIDE SHINDO

University of Illinois at Urbana-Champaign, USA

Tohoku University, Japan

\section{EDITORIAL BOARD}

$\begin{aligned} \text { H. D. BUI } & \text { École Polytechnique, France } \\ \text { J. P. CARTER } & \text { University of Sydney, Australia } \\ \text { R. M. CHRISTENSEN } & \text { Stanford University, USA } \\ \text { G. M. L. GLADWELL } & \text { University of Waterloo, Canada } \\ \text { D. H. HODGES } & \text { Georgia Institute of Technology, USA } \\ \text { J. HUTCHINSON } & \text { Harvard University, USA } \\ \text { C. HWU } & \text { National Cheng Kung University, Taiwan } \\ \text { B. L. KARIHALOO } & \text { University of Wales, UK } \\ \text { Y. Y. KIM } & \text { Seoul National University, Republic of Korea } \\ \text { Z. MROZ } & \text { Academy of Science, Poland } \\ \text { D. PAMPLONA } & \text { Universidade Católica do Rio de Janeiro, Brazil } \\ \text { M. B. RUBIN } & \text { Technion, Haifa, Israel } \\ \text { A. N. SHUPIKOV } & \text { Ukrainian Academy of Sciences, Ukraine } \\ \text { T. TARNAI } & \text { University Budapest, Hungary } \\ \text { F. Y. M. WAN } & \text { University of California, Irvine, USA } \\ \text { P. WRIGGERS } & \text { Universität Hannover, Germany } \\ \text { W. YANG } & \text { Tsinghua University, China } \\ \text { F. ZIEGLER } & \text { Technische Universität Wien, Austria } \\ & \\ \text { PRODUCTION } & \text { contact@ msp.org } \\ \text { SILVIO LEVY } & \text { Scientific Editor }\end{aligned}$

Cover design: Alex Scorpan

Cover photo: Mando Gomez, www.mandolux.com

See http://jomms.org for submission guidelines.

JoMMS (ISSN 1559-3959) is published in 10 issues a year. The subscription price for 2011 is US \$520/year for the electronic version, and \$690/year (+\$60 shipping outside the US) for print and electronic. Subscriptions, requests for back issues, and changes of address should be sent to Mathematical Sciences Publishers, Department of Mathematics, University of California, Berkeley, CA 94720-3840.

JoMMS peer-review and production is managed by EditFLow ${ }^{\circledR}$ from Mathematical Sciences Publishers.

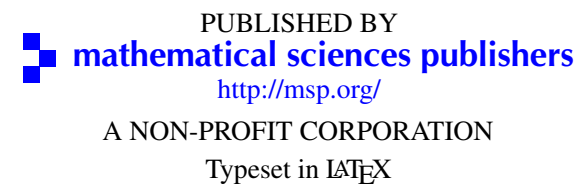

Copyright (C2011 by Mathematical Sciences Publishers 


\section{Journal of Mechanics of Materials and Structures}

\section{Volume 6, No. 7-8}

September-October 2011

\section{Special issue \\ Eleventh Pan-American Congress \\ of Applied Mechanics (PACAM XI)}

Preface

Adair R. Aguiar

949

Influence of specimen geometry on the Portevin-Le Châtelier effect due to dynamic strain aging

for the AA5083-H116 aluminum alloy

Rodrigo Nogueira de Codes and Ahmed Benallal

Dispersion relations for SH waves on a magnetoelectroelastic heterostructure with imperfect

interfaces

J. A. Otero, H. Calas, R. Rodríguez, J. Bravo, A. R. Aguiar and G. Monsivais

Numerical linear stability analysis of a thermocapillary-driven liquid bridge with magnetic stabilization

Yue Huang and Brent C. Houchens

Numerical investigation of director orientation and flow of nematic liquid crystals in a planar 1:4 expansion Pedro a. Cruz, Murilo F. Tomé, IAin W. Stewart and Sean McKee

Critical threshold and underlying dynamical phenomena in pedestrian-induced lateral vibrations of footbridges

Stefano LenCI and LAURA MARCHEgGiani

Free vibration of a simulation CANDU nuclear fuel bundle structure inside a tube

XUAN ZHANG and SHUdONG Yu

Nonlinear dynamics and sensitivity to imperfections in Augusti's model

D. Orlando, P. B. Gonçalves, G. Rega and S. LenCi

Active control of vortex-induced vibrations in offshore catenary risers: A nonlinear normal mode approach

CArlos E. N. MAZzilli and César T. SANCheS

Nonlinear electromechanical fields and localized polarization switching of piezoelectric macrofiber composites

Yasuhide Shindo, Fumio Narita, KoJi SATo and Tomo TAKeda

1089

Three-dimensional BEM analysis to assess delamination cracks between two transversely isotropic materials

Nicolás O. Larrosa, Jhonny E. Ortiz and Adrián P. Cisillino

Porcine dermis in uniaxial cyclic loading: Sample preparation, experimental results and modeling

A. E. Ehret, M. Hollenstein, E. MAzzA and M. Itskov

Analysis of nonstationary random processes using smooth decomposition

Rubens SAMpaio and Sergio Bellizzi

Perturbation stochastic finite element-based homogenization of polycrystalline materials

S. LePage, F. V. Stump, I. H. Kim and P. H. Geubelle

A collocation approach for spatial discretization of stochastic peridynamic modeling of fracture

Georgios I. Evangelatos and POL D. SPANOS 